\title{
Transformation-Enabled Precondition Inference
}

\author{
BISHOKSAN KAFLE \\ IMDEA Software Institute, Madrid, Spain \\ (e-mail: bishoksan.kafle@imdea.org) \\ GRAEME GANGE, PETER J. STUCKEY \\ Faculty of IT, Monash University, Clayton Vic. 3800, Australia \\ (e-mail: $\{$ graeme.gange, peter.stuckey\}@monash.edu) \\ PETER SCHACHTE, HARALD SØNDERGAARD \\ School of Computing and Information Systems \\ The University of Melbourne, Vic. 3010, Australia \\ (e-mail: $\{$ schachte, harald\}@unimelb.edu.au) \\ submitted 1 January 2003; revised 1 January 2003; accepted 1 January 2003
}

\begin{abstract}
Precondition inference is a non-trivial problem with important applications in program analysis and verification. We present a novel iterative method for automatically deriving preconditions for the safety and unsafety of programs. Each iteration maintains over-approximations of the set of safe and unsafe initial states, which are used to partition the program's initial states into those known to be safe, known to be unsafe and unknown. We then construct revised programs with those unknown initial states and iterate the procedure until the approximations are disjoint or some termination criteria are met. An experimental evaluation of the method on a set of software verification benchmarks shows that it can infer precise preconditions (sometimes optimal) that are not possible using previous methods. It is "under consideration for acceptance in TPLP".
\end{abstract}

\section{Introduction}

Precondition analysis infers input conditions that establish runtime properties of interest (for example, a sufficient precondition for safety is a set of initial states, each of which is guaranteed to be safe with respect to given safety properties). Applications include program verification, symbolic execution, debugging, and program comprehension. Derivation of exact preconditions (excluding no good runs and including no bad runs) is undecidable, so the aim is to derive preconditions that are general enough to be useful in practice. We approach the problem by iteratively refining over-approximations of safe and unsafe states. For this, constrained Horn clauses (CHCs) are convenient, as they can model imperative programs and assertions in a uniform way (Peralta et al. 1998, Grebenshchikov et al. 2012, Gurfinkel et al. 2015, De Angelis et al. 2017).

Consider the program in Fig. 1. The left box shows a fragment in $\mathrm{C}$, the right box shows its $\mathrm{CHC}$ representation, encoding reachable states. $\mathrm{C}$ variables are represented by logical variables (capital letters). The clause $c_{1}$ specifies the initial states of the program via the predicate init which is always reachable. Similarly, $c_{2}$ and $c_{3}$ encode the reachability of the while loop via the predicate $w h$. Clause $c_{2}$ states that the loop is reachable if $i n i t$ is 

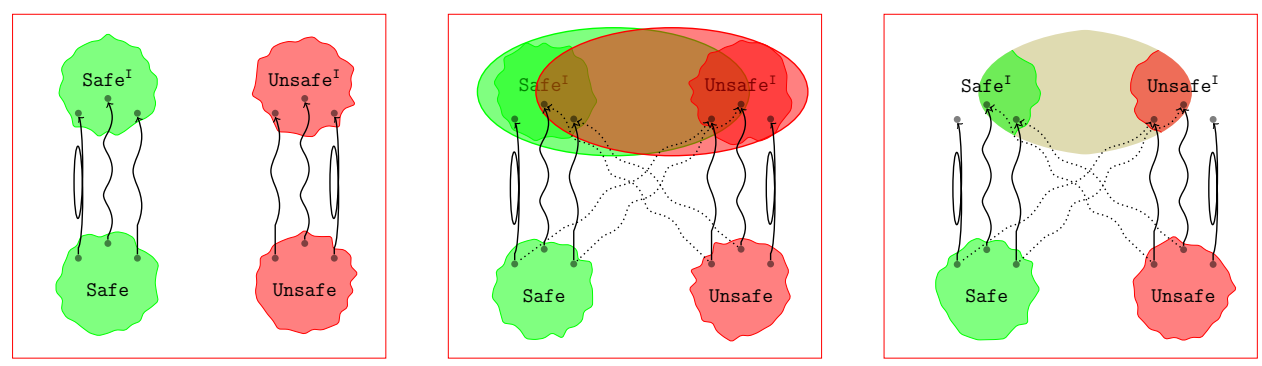

Fig. 2: Precondition inference: Reality (left), initial approximations (middle), one step refinement of approximations using Algorithm 1 (right). Arrows represent preconditions flowing from safe or unsafe final states (bottom) back to corresponding initial states (top).

addressed via forward and backward abstract interpretation, together with constraint specialisation. Using this, one can infer $a \geq 1, b \geq a$ as invariant for predicate wh, as described in Section 3 . Challenge (iii) is addressed by concurrently maintaining and refining approximations of both safe and unsafe states, until the approximations are disjoint or some termination criterion is met.

Fig. 2 sketches the idea. The leftmost panel reflects reality, showing the set of concrete safe and unsafe states, along with the corresponding sets of initial states. Given a program and a description of the sets of interest (safe and unsafe states), precondition analysis infers a set of initial states that lead to these sets of interest. Or rather, it finds over-approximations of the initial sets of states - so these may overlap. Overapproximations are shown as ellipses in the middle panel, coloured appropriately for safe and unsafe initial states. Because of approximation, there may be witness traces from the left ellipse to Unsafe (the dotted arrows) and vice versa. The algorithm aims to reduce the ellipses progressively to the point where the ellipses no longer overlap. A single refinement step that focuses our attention only on the intersection is illustrated in the rightmost panel.

Our work builds upon the transformation-guided framework of Kafle et al. (2018) and incorporates a number of program transformations known from the literature, including

- Partial evaluation (Jones et al. 1993): PE wrt. a goal specialises a program for the given goal; preserving only those derivations that are relevant for deriving the goal.

- Constraint Specialisation (Kafle and Gallagher 2017a) via forward and backward abstract interpretation (Bakhirkin and Monniaux 2017): This strengthens constraints in clauses by exploiting generated invariants, while preserving derivations of a goal. The effect is to prune paths that are not relevant for deriving the goal.

- Trace Elimination (Kafle and Gallagher 2017b): This eliminates a set of traces from a program while preserving the rest of traces and serves to refine a program.

Our contribution is to combine these techniques into an iterative framework that can control the quality of the preconditions for both safety and unsafety. Kafle et al. (2018) iteratively apply $\mathrm{CHC}$ transformations to a program wrt. error, approximating the unsafe states whose complement yields sufficient preconditions for the safety. A disadvantage of this is the blind refinement of unsafe states without knowing its frontier with the safe 
states. This misses opportunities to avoid redundant computation as well as to guide the refinement process at an early stage. We extend that work in a number of directions:

- We model both the safe and unsafe program states, and refine them concurrently, allowing us to derive preconditions for both safety and unsafety. In addition, we show how to derive preconditions for both safety and unsafety either from the original program or one obtained via a sequence of transformations (\$3).

- We present an iterative algorithm to refine approximations of these states, each iteration focusing only on states yet to be shown safe or unsafe (the intersection of safe and unsafe over-approximations), thus reducing the search space. It has refined termination criteria to control precision and detect optimality of the preconditions.

- Reasoning simultaneously about safe and unsafe states allows us to derive precondition for non-termination as a complement of necessary preconditions for safety and unsafety (\$4).

- Evaluation shows that we not only infer non-trivial preconditions in slightly more cases but also infer optimal ones in some cases (\$5).

\section{Preliminaries}

An atom is a formula $p(\mathbf{x})$ where $p$ is a predicate symbol and $\mathbf{x}$ a tuple of arguments. A constrained Horn clause $(\mathrm{CHC})$ is a first-order formula written as $p_{0}\left(\mathbf{x}_{0}\right) \leftarrow$ $\varphi, p_{1}\left(\mathbf{x}_{1}\right), \ldots, p_{k}\left(\mathbf{x}_{k}\right)$ following Constraint Logic Programming (CLP) standard, where $\varphi$ is a finite conjunction of quantifier-free constraints on variables $\mathbf{x}_{i}$ with respect to some constraint theory $\mathbb{T}, p_{i}\left(\mathbf{x}_{i}\right)$ are atoms. A constrained fact is a clause of the form $p_{0}\left(\mathbf{x}_{0}\right) \leftarrow \varphi$, where $\varphi$ is a constraint. We assume the theory $\mathbb{T}$ is equipped with a decision procedure and a projection operator, and that it is closed under negation.

The notation $\left.\varphi\right|_{V}$ represents the constraint formula $\varphi$ projected onto variable set $V$ and $\varphi \models_{\mathbb{T}} \psi$ (or equivalently $\models_{\mathbb{T}} \varphi \rightarrow \psi$ ) to represent $\varphi$ entails $\psi$ over $\mathbb{T}$. Similarly, we write $P \vdash_{\mathbb{T}} A$ when an atom $A$ is derivable from the program $P$ wrt. the theory $\mathbb{T}$.

We use CHCs to encode control flow of C-like programs. Two special predicates, exit0 and error, encode safe and unsafe (error) states, respectively. So exit0 indicates a normal return; error indicates abnormal termination. The predicate init encodes the set of initial states. We assume users specify all states of interest by appropriate constructs provided by the language (e.g., assert(c), return $\langle n\rangle$ of C). States not specified by the user (e.g., buffer-overflow, floating point exceptions) are not taken into account while generating CHCs. Hence correctness of the preconditions depends on the user specified set of states.

From here on, when talking about a program, we refer to its CHC representation.

Definition 1 (AND-tree (Gallagher and Lafave 1996))

An $A N D$-tree for a CHC program $P$ is a tree whose nodes are labelled as follows.

1. Each non-leaf node corresponds to a (renamed) clause in $P$ of the form $A \leftarrow$ $\varphi, A_{1}, \ldots, A_{k}$ where $k>0$. The clause is renamed so that any variables not appearing in $A$ are fresh. The node is labelled by $(A, \varphi)$. The node has $k$ child nodes where the $i^{\text {th }}$ child corresponds to a clause in $P$ of the form $A_{i} \leftarrow \varphi_{i}, B_{i}$ where $B_{i}$ may be empty and is labelled by $\left(A_{i}, \varphi_{i}\right)$.

2. Each leaf node corresponds to a (renamed) clause in $P$ of the form $A \leftarrow \varphi$ and is labelled as $(A, \varphi)$. 
Given an AND-tree $t$, constr $(t)$ is the conjunction of the constraints appearing in the tree. The tree $t$ is feasible if and only if constr $(t)$ is satisfiable over $\mathbb{T}$.

Definition 2 (Initial clauses and nodes)

Let $P$ be a program with a distinguished predicate $p^{I}$ which we call the initial predicate. The constrained facts $\left\{\left(p^{I}(\mathbf{x}) \leftarrow \theta\right) \mid\left(p^{I}(\mathbf{x}) \leftarrow \theta\right) \in P\right\}$ are the initial clauses of $P$. Let $t$ be an AND-tree for $P$. A node labelled by $p^{I}(\mathbf{x}) \leftarrow \theta$ is an initial node of $t$. We extend the term "initial predicate" and use the symbol $p^{I}$ to refer also to renamed versions of the initial predicate that arise during clause transformations.

In Fig. 1 the initial predicate is init and the initial clause is init(A,B).

\section{Program transformations and preconditions}

We now show how to find preconditions for safety and unsafety for a program (original or obtained via transformation). We limit attention to sets of clauses for which every AND-tree for exit0 and error (whether feasible or infeasible) has at least one initial node.

Definition 3 (Program with initial states $\varphi\left(P_{\varphi}^{I}\right)$ and replaced states $\varphi\left(P_{\varphi}^{R}\right)$ )

Let $P$ be a program and $\varphi$ a constraint over $\mathbb{T}$. Let $P_{\varphi}^{I}$ be the clauses obtained from $P$ by replacing the initial clauses $\left\{\left(p^{I}(\mathbf{x}) \leftarrow \theta_{i}\right) \mid 1 \leq i \leq k\right\}$ by $\left\{\left(p^{I}(\mathbf{x}) \leftarrow \varphi \wedge \theta_{i}\right) \mid 1 \leq i \leq k\right\}$. Similarly, let $P_{\varphi}^{R}$ be the set of clauses obtained from $P$ by replacing the initial clauses $\left\{\left(p^{I}(\mathbf{x}) \leftarrow \theta_{i}\right) \mid 1 \leq i \leq k\right\}$ by $\left\{\left(p^{I}(\mathbf{x}) \leftarrow \varphi\right)\right\}$.

Definition 4 (Necessary/sufficient precondition for safety)

Let $P$ be a program and $\varphi$ a constraint over $\mathbb{T}$. Then

- a constraint $\varphi$ is a necessary precondition (NP) for the safety of $P$ if $P \vdash_{\mathbb{T}}$ exit0 entails $P_{\varphi}^{R} \vdash_{\mathbb{T}}$ exit0. In words, $\varphi$ (possibly true) is an over-approximation of the set of initial states of $P$ that can reach exit0.

- a constraint $\psi$ is a sufficient precondition (SP) for the safety of $P$ if $P_{\psi}^{I} \forall_{\mathbb{T}}$ error. In words, $\psi$ (possibly false) is an under-approximation of the set of initial states of $P$ that cannot reach error.

Thus an SP for safety is a constraint that suffices to block derivations of error (given we assume clauses for which $p^{I}$ is essential for any derivation of error). In practice we would like to consider SP for safety as a constraint that allows derivations of exit0 and blocks derivations of error. We define NP $\left(\operatorname{nec}_{\mathrm{u}}(P)\right)$ and SP $\left(\operatorname{suf}_{\mathrm{u}}(P)\right)$ for unsafety analogously. In the following, we show how an NP and an SP can be derived from a set of clauses.

Definition 5 (NP extracted from CHC program $P$ )

Let $P$ be a set of clauses encoding reachable states of a program. The formula

$$
\bigvee\left\{\theta \mid\left(p^{I}(\mathbf{x}) \leftarrow \theta\right) \in P\right\} .
$$

is an NP for both safety and unsafety. We refer to it as $\operatorname{nec}_{\mathbf{s}}(P)$ when talking about safety and as $\operatorname{nec}_{\mathrm{u}}(P)$ when talking about unsafety.

The reason why $\operatorname{nec}_{\mathbf{s}}(P)$ is an NP for safety is that any feasible AND-tree for $P$ must use at least one initial clause of $P$ and so the disjunction of constraints from the initial clauses (although imprecise) is a sound NP for the safety, as well as the unsafety, of $P$. Using 


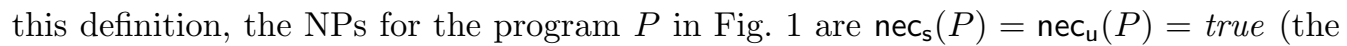
set of initial states of the program). Given NPs for a program $P$, we can find sufficient preconditions for the safety and unsafety as follows.

Definition 6 (SP extracted from $C H C$ program $P$ )

Let $P$ be a set of clauses encoding reachable states of a program. We define

$$
\begin{aligned}
& \operatorname{suf}_{\mathrm{s}}(P)=\operatorname{nec}_{\mathrm{s}}(P) \wedge \neg \operatorname{nec}_{\mathrm{u}}(P) \\
& \operatorname{suf}_{\mathrm{u}}(P)=\operatorname{nec}_{\mathrm{u}}(P) \wedge \neg \operatorname{nec}_{\mathrm{s}}(P)
\end{aligned}
$$

The former is a sufficient precondition for safety, the latter for unsafety, of $P$. In the sequel, we represent necessary and sufficient preconditions by Greek letters $\varphi$ and $\psi$, respectively, possibly with subscript $u$ for unsafety and $s$ for safety.

Let $\varphi_{c}$ be $\operatorname{nec}_{\mathrm{s}}(P) \wedge \operatorname{nec}_{\mathrm{u}}(P)$, denoting the shared region between the (approximate) safe and unsafe states. A precondition is separating (optimal) if $\varphi_{c}$ is unsatisfiable (the safe and unsafe regions are separated). Then we have $\operatorname{suf}_{\mathrm{s}}(P)=\operatorname{nec}_{\mathbf{s}}(P) \operatorname{since~nec}_{\mathrm{s}}(P) \models_{\mathbb{T}}$ $\neg \mathrm{nec}_{\mathrm{u}}(P)$. That is, necessary and sufficient conditions are the same for the validity of the assertion. Analogously, we have $\operatorname{suf}_{\mathrm{u}}(P)=\operatorname{nec}_{\mathrm{u}}(P)$.

The shared region characterised by $\varphi_{c}$ indicates imprecision of over-approximationswhich we attempt to reduce as much as possible. We achieve this reduction as follows:

1. Construct a revised program $P_{\varphi_{c}}^{I}$ (Def. 3 from $P$ focusing only on the shared region such that its SP is a valid SP for $P$ (Lemma 3.1).

2. Shrink either of the regions via iterative strengthening of the initial clauses of the program from where necessary preconditions are derived.

For this we utilize well-known CHC transformations, from the literature on CLP and Horn clause verification, as outlined below.

\section{Proposition 3.1}

Let $P$ be a program, $\varphi$ a constraint and $P_{\varphi}^{I}$ as defined in Def. 3 . Let $\psi$ be any SP for the safety (unsafety) of $P_{\varphi}^{I}$. Then $\psi$ is also an SP for the safety (unsafety) of $P$.

1. Partial Evaluation (PE). The PE algorithm we use (Gallagher 2019) produces a polyvariant specialisation, that is, a finite number of versions of each predicate, which is essential for deriving disjunctive information as well as for refining the control-flow of the program (Doménech et al. 2019). The result of applying PE to the example program in Fig. 1 wrt. error and exit0 is shown in Fig. 3 . For details we refer to Gallagher (2019). A key point is that, owing to polyvariant specialisation, init and wh are split into two different versions, leading to more precise preconditions as in Eq. (1) (using Def. 5).

$$
\begin{aligned}
& \varphi_{u}=(\mathrm{B}<0 \wedge \mathrm{A} \leq 0) \vee \mathrm{A} \geq 1 \equiv \mathrm{B}<0 \vee \mathrm{A} \geq 1 \\
& \varphi_{s}=(\mathrm{B} \geq 0 \wedge \mathrm{A} \leq 0) \vee(\mathrm{A} \geq 1 \wedge \mathrm{B} \geq 0) \equiv \mathrm{B} \geq 0
\end{aligned}
$$

2. Constraint Specialisation (CS). CS (Kafle and Gallagher 2017a) of program $P$ wrt. goal $A$ specialises each constraint $\varphi$ in a clause of $P$ to a constraint $\varphi \wedge \psi$ while preserving the derivation of $A$. Fig. 4 shows application of CS to Fig. 3 (left) wrt. error, and to Fig. 3 (right) wrt. exit0. Note that only clauses that got specialised are shown, and the newly derived constraints are underlined for readability. The underlined constraints in Fig. 4 


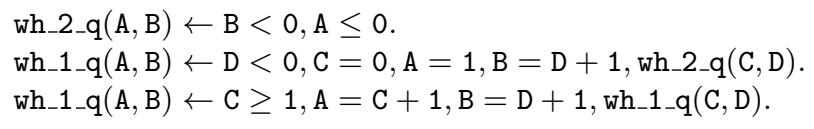

Fig. 5: Excerpt of query clauses corresponding to the program in Fig. 3 (left) starting from the goal (query) error; the suffix q denotes a query predicate.

Lemma 3.2 (Adapted from Kafle et al. (2018) for AND-tree of exit0)

Let $P^{\prime}$ be the result of eliminating a feasible AND-tree $t$ for exit0 (resp. error) from $P$. Then $\operatorname{nec}_{\mathbf{s}}(P)=\operatorname{nec}_{\mathbf{s}}\left(P^{\prime}\right) \vee \theta_{t}\left(\operatorname{resp} . \operatorname{nec}_{\mathbf{u}}(P)=\operatorname{nec}_{\mathbf{u}}\left(P^{\prime}\right) \vee \theta_{t}\right)$, where $\theta_{t}$ is a constraint extracted from $t$ (Def. 7).

Observe that the elimination of feasible traces acts as program decomposition. Transformations such as PE, CS and TE (when used to remove infeasible trees) not only preserve the goal but also the initial clauses. This allows us to construct a sequence of clauses $P_{0}, P_{1}, \ldots, P_{m}$ where $P=P_{0}$ and each element of the sequence is more specialised than its predecessor wrt. derivations of exit0 (error). As a consequence, the NPs are more precise. We write $P \Longrightarrow_{A} P^{\prime}$ when $P^{\prime}$ is a goal-preserving transformation of $P$ wrt. an atom $A$, that is, $P \models A$ iff $P^{\prime} \models A$. TE (eliminating feasible trees) is a little different, in that it does not preserve the goal. We abuse the notation and write $P \Longrightarrow_{t_{A}} P^{\prime}$ for transformation of $P$ by eliminating a feasible tree rooted at $A$, yielding $P^{\prime}$. Lemma 3.2 ensures soundness of preconditions in this case.

Let us now wrap these transformations and their combinations. Let tr and tr-seq be any functions satisfying the following:

$$
\begin{aligned}
& \operatorname{tr}_{A}\langle P, \varphi\rangle= \begin{cases}\left\langle P^{\prime}, \varphi\right\rangle & \text { where } P \Longrightarrow_{A} P^{\prime} \\
o r & \\
\left\langle P^{\prime}, \varphi^{\prime}\right\rangle & \text { where } \left.P \Longrightarrow_{t_{A}} P^{\prime} \text { and } \varphi^{\prime}=\varphi \vee \theta_{t_{A}} \text { (Def. } 7\right)\end{cases} \\
& \operatorname{tr}^{-\operatorname{seq}_{A}}\langle P, \varphi\rangle=\operatorname{tr}_{A}^{n}\langle P, \varphi\rangle \text { for } n \geq 1
\end{aligned}
$$

where $f^{n}=f^{n-1} \circ f$, with $f^{1}=f$. tr-seq allows us to combine the above transformations in any order and Proposition 3.3 allows us to derive more precise preconditions.

\section{Proposition 3.3}

Let $P$ be a program, $\left\langle P_{s}, \varphi_{s}\right\rangle=\operatorname{tr}-$ seq $_{\text {exito }}\langle P$, false $\rangle$. Then $\models_{\mathbb{T}}\left(\operatorname{nec}_{\mathbf{s}}\left(P_{s}\right) \vee \varphi_{s}\right) \rightarrow \operatorname{nec}_{\mathbf{s}}(P)$. Similarly, if $\left\langle P_{u}, \varphi_{u}\right\rangle=$ tr-seq error $_{\text {r }}\langle P$, false $\rangle$, then $\models_{\mathbb{T}}\left(\operatorname{nec}_{\mathrm{u}}\left(P_{u}\right) \vee \varphi_{u}\right) \rightarrow \operatorname{nec}_{\mathrm{u}}(P)$.

\section{An algorithm for precondition inference}

We now give an algorithm for computing SPs for safety and unsafety as Algorithm 1 based on the transformations described previously. Input is a set of CHCs (involving clauses for exit0, error and init) and a sequence of transformations tr-seq. Output is a pair of SPs for safety and unsafety. The SPs $\psi_{s}$ and $\psi_{u}$ are initialised to false. The algorithm aims to weaken these SPs as far as it can. $\varphi_{\text {old }}$ keeps track of the set of initial states that are yet to be proven safe or unsafe. $P_{s}$ and $P_{u}$ respectively keep track of the transformations of $P$ with respect to exit0 and error.

In the algorithm, the following operations are carried out in an iterative manner and possibly in parallel (within the while loop). The instructions on two sides of the boxes can 


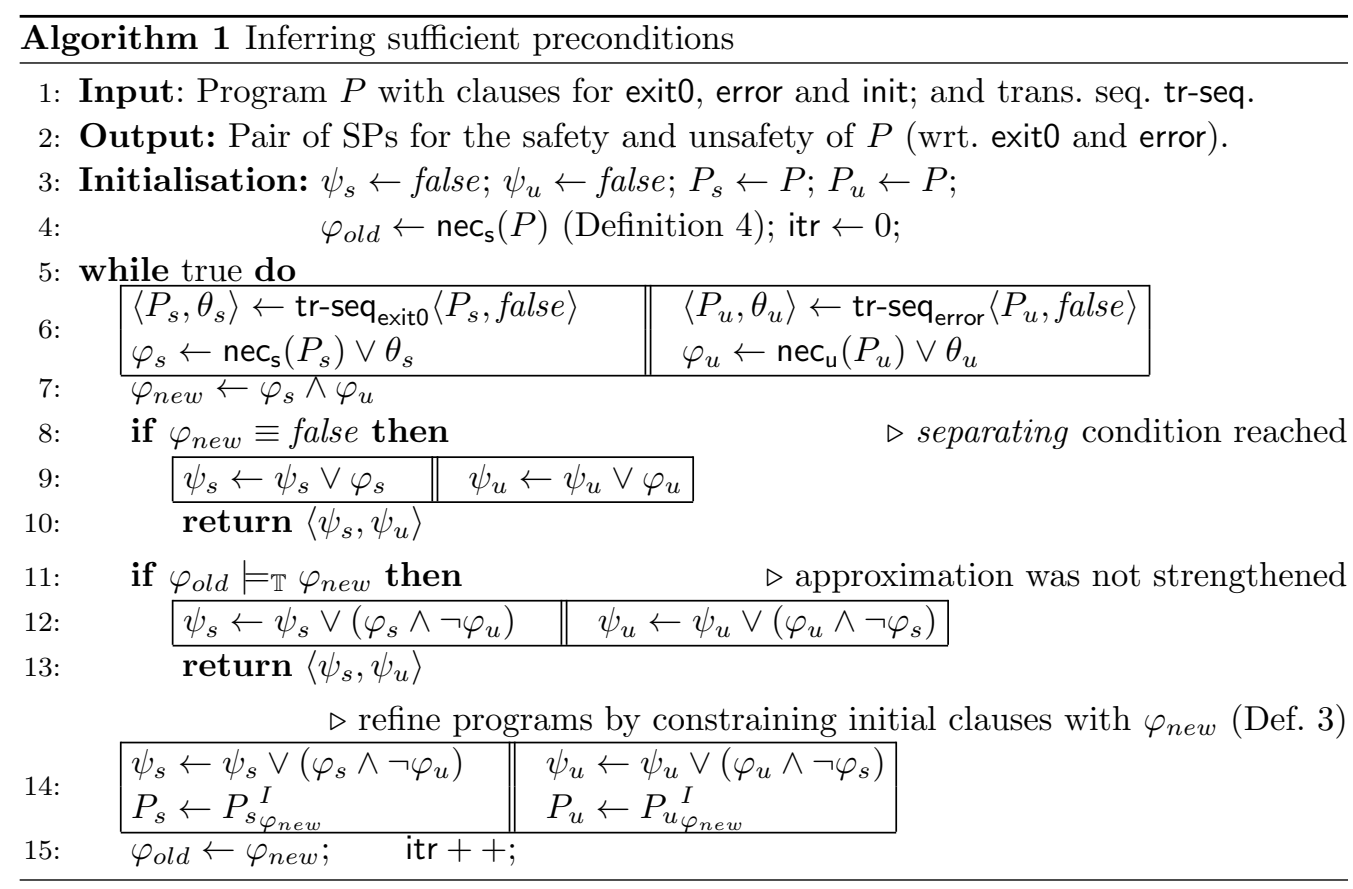

be executed in parallel. One or more of the transformation of $P_{s}$ and $P_{u}$ with respect to exit0 and error, respectively, are carried out and the NPs are extracted from the resulting programs (line 6). The algorithm terminates and returns an SP if the conjunction of these NPs is unsatisfiable (line 10, separating) or it is not stronger (wrt. $\models_{\mathbb{T}}$ ) than $\varphi_{\text {old }}$ (line 13). Otherwise, the algorithm iterates with revised programs obtained by constraining their initial clauses with the conjunction $\varphi_{\text {new }}$ (line 14). For this, $\varphi_{\text {new }}$ needs to be converted to DNF that may blow up the number of resulting initial clauses. In our experiments, the largest size of DNF discovered was 11. Even if all transformations in the algorithm terminate, it may still not terminate since $\varphi_{\text {new }}$ can infinitely be decreased. But it makes a progress, that is, it explores a strictly smaller set of initial states in each iteration that have not yet been known safe or unsafe. This is formalised in Proposition 4.1. Observe that each iteration computes valid SPs for the safety and unsafety of the original program (e.g., line 14) and combines them disjunctively with the previous SPs. Proposition 4.2 ensures that the combination yields valid SPs for the original program.

Proposition 4.1 (Progress and Termination of Algorithm 1)

Algorithm 1 either progresses or terminates.

Proposition 4.2 (Composing Preconditions)

Let $\Phi$ be a set of formulas such that each $\varphi \in \Phi$ is an SP for (un)safety of $P$. Then $\bigvee \Phi$ is also an SP for (un)safety of $P$.

Proposition 3.3 ensures the correctness of the transformations sequence, Proposition 3.1 ensures that the precondition of $P_{\varphi}^{I}$ is also that of $P$, and Proposition 4.2 allows us to combine the preconditions derived in the separate iterations. Together they ensure the soundness of Algorithm 1 . 
whereas Seghir and Schrammel (2014) ignore them, as do we. However, the modelling of safe and unsafe terminating states and their over-approximations allow us to reason about a limited form of non-termination as suggested by Popeea and Chin (2013): Any input state that is neither in the over-approximation of safe nor unsafe leads to nontermination assuming we model all terminating (un)safe states.

We demonstrate this with Fig. 7. The program does not terminate if $a \in[0,10]$. We derive $\varphi_{u}=$ $\mathrm{a}<0$ and $\varphi_{s}=\mathrm{a} \geq 11$ as NPs. Thus the condition satisfying $\neg\left(\varphi_{u} \vee \varphi_{s}\right)$, that is, a $\in[0,10]$ is a sufficient precondition for non-termination (which happens to be the exact condition in this case). It is obtained as a byproduct of our method; we leave the primary analysis of non-termination for future work.

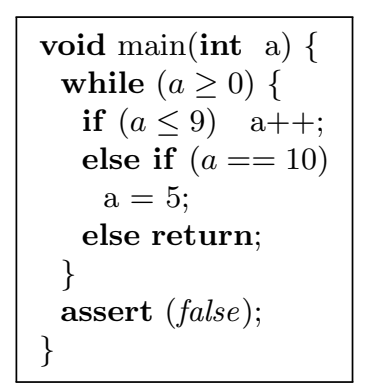

Fig. 7: Non-termination

\section{Experimental evaluation}

Since we model both the safe and unsafe program states and successively refine them to be able to detect separating or more precise preconditions for the safety and unsafety of programs, the experiments were designed to better answer the following questions.

- Q1. Does the algorithm allow us to derive separating preconditions in practice?

- Q2. Does refinement allow us to derive more non-trivial or separating preconditions?

- Q3. How does our approach compare to that of state-of-the-art tools for precondition inference in terms of the quality of the preconditions and performance?

Experimental Setup. We implemented Algorithm 1 (a sequential version) in PI-HorN ${ }^{1}$ The implementation applies the sequence TE॰CSoPE. The tool is written in Ciao Prolog (Hermenegildo et al. 2012), using PPL (Bagnara et al. 2008) and Yices2 (Dutertre 2014). Input is a set of CHCs, with exit0, error and init as distinguished predicates. PI-HoRN outputs a pair of SPs for safety and unsafety and are classified as: (i) optimal: the precondition is both necessary and sufficient (exact); (ii) non-trivial: the precondition is different from false (but not optimal) and (iii) trivial: the precondition is false.

Experiments were conducted on a MacBook Pro, running OSX 10.11 with 16GB memory and $2.7 \mathrm{GHz}$ Intel Core i5 processor. We tested our approach with 261 integer programs (available from https://github.com/bishoksan/PI-Horn/tree/master/ benchmarks) sourced as follows: (i) 150 integer programs from the loop (69) and recursive (81) subcategories of the Integers and Control Flow category of SV-COMP (Beyer 2021); (ii) 83 programs from the DAGGER (Gulavani et al. 2008) and TRACER tools (Jaffar et al. 2012) and (iii) 28 programs from the literature on precondition inference and backwards analysis (Bakhirkin et al. 2014, Miné 2012, Moy 2008, Bakhirkin and Monniaux 2017; Cassez et al. 2017). We are unable to include some benchmarks used by Kafle et al. (2018) owing to unavailability of their $\mathrm{C}$ sources which are needed to model the exit states. Benchmark set (i) was designed for verification competitions, (ii)

1 "Precondition Inferrer for Horn clauses", available at https://github.com/bishoksan/PI-Horn 


\begin{tabular}{|r|r|r|r|r|r|r|r|r|}
\hline iter & opt & ntS (Sw) & ntU (Uw) & ntSU & tSU & $\begin{array}{l}\text { PI-HORN } \\
\text { total/iter }\end{array}$ & $\begin{array}{c}\text { WP-RAHFT } \\
\text { total/iter }\end{array}$ \\
\hline 0 & 58 & 0 & $(0)$ & $0(0)$ & 0 & 0 & 58 & 197 \\
\hline 1 & 87 & 9 & $(7)$ & $5(3)$ & 2 & 20 & 99 & 20 \\
\hline 2 & 21 & $20(15)$ & $7(2)$ & 5 & 0 & 43 & 0 \\
\hline 3 & 5 & 6 & $(3)$ & $3(0)$ & 3 & 0 & 11 & 0 \\
\hline 4 & 2 & 3 & $(1)$ & $2(0)$ & 2 & 0 & 5 & 1 \\
\hline 5 & 2 & 0 & $(0)$ & $0(0)$ & 0 & 0 & 2 & 0 \\
\hline 6 & 1 & 0 & $(0)$ & $0(0)$ & 0 & 0 & 219 & 218 \\
\hline \#total & 176 & $38(26)$ & $17(5)$ & 12 & 20 & 0 & 2 & 0 \\
\hline
\end{tabular}

Table 1: Experimental results on 261 programs, with a timeout of 300 seconds

and (iii) to demonstrate particular tools and techniques. We adapt these $\mathrm{C}$ programs for precondition inference as follows. They are translated to CHCs of the required form based on specialisation approach of De Angelis et al. (2017) using VeriMap (De Angelis et al. 2014). We then replace the generated init $(\mathbf{x}) \leftarrow \varphi(\mathbf{x})$ clause by $\operatorname{init}(\mathbf{x}) \leftarrow$ true. This allows analyses to infer preconditions in terms of $\mathbf{x}$, starting from an unrestricted set of initial clauses.

Results and Discussion. Table 1 shows the results. The columns 2-7 show results for PI-HoRN and the last column for WP-RAHFT (Kafle et al. 2018). The first column iter indicates the number of refinement iterations for both. The columns show opt (\# programs with separating preconditions), ntS (Sw) (\# programs with non-trivial SPs for safety excluding separating cases, and, in parentheses, the difference with trivial SPs for unsafety), ntU (Uw) (same, for unsafety), ntSU (\# programs with non-trivial SPs for both safety and unsafety), tSU (\# programs with trivial SPs for both safety and unsafety), total/iter (\# programs with non-trivial (either for safety or unsafety) plus separating SP per iteration), WP-RAHFT total/iter (\# programs with non-trivial SP per iteration for WP-RAhFT). For example, the entry 9 (7) in column 3 indicates that there were 9 non-trivial SPs for safety, of which 7 had trivial SPs for unsafety. In other words, the number in the parentheses counts the pairs of the form $\langle\mathrm{ntS}, \mathrm{tU}\rangle$ where $\mathrm{tU}$ means trivial precondition for unsafety. Each row corresponds to an iteration and contains the number of instances solved in that iteration, excluding those solved in the previous.

The results answer Q1 and Q2 positively. PI-HoRN infers non-trivial preconditions for $83 \%$ and optimal ones for $67 \%$ of the programs. Interestingly, it infers optimal preconditions for 58 programs owing to specialisation transformations alone (see row \#1, iter 0 ), whereas it infers non-trivial preconditions for 99 programs (of which 87 are optimal) after the first refinement. More non-trivial preconditions are derived when refinement progresses. This indicates that both the preprocessing and refinement significantly increase the number of optimal (non-trivial) cases. However, for 63 out of 261 programs, refinement did not progress towards optimality (that is, it did not further shrink the approximations of safe and unsafe states). We also observe that it timed out on $9 \%$ and failed to infer any meaningful preconditions for $8 \%$.

As for Q3 we could not meaningfully compare our tool against the work of Seghir and Schrammel (2014), or Bakhirkin et al. (2014), in the first case because of tool issues (discovered together with the authors), and a lack of automation (confirmed by the authors via email) for the second. We do compare with WP-RAHFT (Kafle et al. 2018), 
but note that, while some of the components of the tools are identical, the results are not directly comparable. The success of PI-HoRN depends on its ability to refine both the approximations simultaneously unlike WP-RAHFT. For example, we might obtain a tight bound $\varphi$ for safe states but if the approximation of unsafe states is $\psi$ such that $\varphi \models_{\mathbb{T}} \psi, \psi \not \equiv$ true then PI-HoRN returns trivial SP for safety whereas WP-RAHFT returns non-trivial. On the other hand, WP-RAHFT cannot detect optimality and does not derive preconditions for unsafety. The two tools provide almost the same number of programs with non-trivial SPs (219 vs 218), but they differ in the quality (e.g., optimality) of preconditions. Since WP-RAHFT cannot detect optimality, limited information about it can be obtained by checking suf $\mathrm{PI}_{\text {I-HORN }} \models_{\mathbb{T}}$ suf $_{\mathrm{WP} \text {-RAHFT }}$ on all those instances that are known to be optimal, where suf $\mathrm{x}_{\mathrm{x}}$ represents the sufficient precondition derived by the tool $x$. From this, we report that WP-RAHFT derives optimal preconditions for 58 programs (\# of successful checks), far less than PI-HoRN (176). Every refinement yields improvements for PI-HoRN but refinement beyond the second yields negligible improvements for WP-RAHFT. This affirms that focusing attention on the intersection of approximations of safe and unsafe states is a good refinement strategy and shows the benefit of concurrently approximating these states. Thanks to the refined termination criteria of PI-HonN that the average time in seconds per instance is 30.72 (22 timeouts), while for WP-RAHFT it goes from 28.14 (iter 1, 20 timeouts) to 41.86 (iter 6, 30 timeouts). In summary for Q3, PI-HoRN infers better preconditions than WP-RAHFT and shows reasonable performance.

\section{Related work}

Over-approximation techniques (forward/backward abstract interpretations or their combination (Cousot and Cousot 1992, Cousot et al. 2013, Bakhirkin and Monniaux 2017)) inherently derive NPs, and complementation supplies SPs at a cost of precision (due to approximation of the complement). Howe et al. (2004) use a pseudo-complemented domain (Pos) domain (Marriott and Søndergaard 1993) to infer SPs; Bakhirkin et al. (2014) exchange an abstract complement operation for abstract logical subtraction. Our method neither assumes an abstract domain is (pseudo-) complemented nor apply complementation of abstract elements during analysis. It applies to any abstract domain, and complementation is carried out externally to abstract interpretation, storing the result as a formula without any loss of precision.

Little work has been done that inherently computes SPs without complementation. The notable exception is the work by Miné (2012), who designs all required purposebuilt backward transfer functions for intervals, octagons and convex polyhedra domains. The downside is that the purpose-built operations, including widening, can be rather intricate and require substantial implementation effort. Moy (2008) employs weakestprecondition reasoning and forward abstract interpretation to generalise conditions at loop heads to infer SPs. The derived conditions offer limited use except for a theorem prover. Our method, on the other hand, uses standard techniques and off-the-shelf tools. Output from PI-HoRN can be consumed by other analysis and verification tools.

In a verification context, the dual-analysis approach of Popeea and Chin (2013) uses over-approximations, as we do, to concurrently infer NPs for safety and unsafety; from that, SPs are derived. No attempt is made to weaken those preconditions (by refining the 
approximations or focusing the analysis, as we do); we suspect such SPs are overly strong. Dillig et al. (2013) use Hoare style reasoning with abduction iteratively, to infer loop invariants that are sufficient to show validity of assertions. The success of their method relies on guessing good abducibles whereas computing precise inductive invariants is too hard to achieve for realistic programs (due to undecidability).

Program transformation approaches that preserve the goal can be used to derive preconditions, as our approach. These include the forward/backward iterative specialisation by De Angelis et al. (2014), for verifying program properties. The transformation approach uses a constraint generalisation instead of abstract interpretation. Similarly, the multivariant top-down analyzer by Puebla and Hermenegildo (1999; Muthukumar and Hermenegildo (1990) produces polyvariant specializations, as in the classical algorithms, performing backwards analysis using abstract interpretation. These methods are complementary to ours and we leave a comparative study with our method for future work.

Seghir and Schrammel (2014) use a CEGAR approach to derive exact necessary and sufficient preconditions for safety. Like us, they model safe and unsafe states of a program and refine their approximations until they are disjoint. Their algorithm may diverge due

to (i) the lack of a suitable generalisation of the counterexamples (an inherent limitation of CEGAR) and (ii) the termination condition (disjointness) that is too hard to achieve for realistic programs (due to undecidability). Padhi et al. (2016) attempt to derive optimal preconditions using machine learning approaches. The success of their approach relies on learning good heuristics to separate good runs from bad runs. We, in contrast, use abstract interpretation and program transformation, so each step of the algorithm terminates and a sound precondition can be derived from the resulting programs. Besides, optimality is not the end goal for us and it is a by-product of precision refinement.

The work of Kafle et al. (2018) is orthogonal to those above, combining a range of established techniques such as abstract interpretation, CEGAR and program transformations in a profitable way. The iterative nature of their approach allows them to derive more precise preconditions for safety, however the termination criterion, the maximum number of iterations supplied by the user, is rather weak and cannot be used to optimality of the preconditions. The current work offers several advantages. We model both safe and unsafe states that enables us to detect optimality and also infer NP and SP for both safety and unsafety. In addition, it allows reasoning about a limited form of non-termination and provides more refined termination criteria. Unlike many methods in the literature (Seghir and Schrammel 2014, Bakhirkin et al. 2014), our method can uniformly handle programs with procedures and recursive programs.

\section{Concluding remarks}

We have presented an iterative method for automatically deriving sufficient preconditions for the safety and unsafety of programs. It maintains over-approximations of the set of safe and unsafe initial states. Each iteration of the algorithm considers only states that are common to these approximations as they are yet to be classified as safe or unsafe. The method terminates when the common set of states is empty or it fails to shrink in successive iterations. In experiments, the method generated separating preconditions in $67 \%$ of test cases and solved problems which fail to resolve using only approximation of unsafe states (as done in previous work). Owing to over-approximation, the sufficient precondi- 
tions may include some non-terminating states, which hinders the derivation of optimal preconditions. Our method can only infer preconditions that are expressible as boolean combinations of (quantifier free) linear integer constraints and the prototype implementation mostly ignores simplification of preconditions, possibly leaving redundancies. In future work, we intend to augment our method with non-termination analysis, extend it to infer quantified preconditions and work on simplifying the preconditions.

\section{Acknowledgements}

We thank John Gallagher and three anonymous reviewers whose suggestions helped improve the paper. We are also grateful for help with the use of VeriMap for C to CHC translation, provided to us by Emanuele De Angelis. Bishoksan Kafle has been partially funded by the Spanish Ministry of Research, Science and Innovation, grant MICINN PID2019-108528RB-C21 ProCode and Madrid P2018/TCS-4339 BLOQUES-CM.

\section{References}

Bagnara, R., Hill, P. M., And Zaffanella, E. 2008. The Parma Polyhedra Library: Toward a complete set of numerical abstractions for the analysis and verification of hardware and software systems. Sci. Comput. Program. 72, 1-2, 3-21.

Bakhirkin, A., Berdine, J., ANd Piterman, N. 2014. Backward analysis via over-approximate abstraction and under-approximate subtraction. In SAS'14. LNCS, vol. 8723. Springer, 34-50.

Bakhirkin, A. And Monniaux, D. 2017. Combining forward and backward abstract interpretation of Horn clauses. In $S A S^{\prime} 17$. LNCS, vol. 10422. Springer, 23-45.

BEYER, D. 2021. Software verification: 10th comparative evaluation (SV-COMP 2021). In TACAS 2021, J. F. Groote and K. G. Larsen, Eds. LNCS. Springer, 401-422.

Beyer, D., Henzinger, T. A., Majumdar, R., and Rybalchenko, A. 2007. Path invariants. In PLDI, J. Ferrante and K. S. McKinley, Eds. ACM, 300-309.

Cassez, F., Jensen, P. G., and Larsen, K. G. 2017. Refinement of trace abstraction for real-time programs. In Reachability Problems. LNCS, vol. 10506. Springer, 42-58.

Codish, M. And SøndergaARd, H. 2002. Meta-circular abstract interpretation in Prolog. In The Essence of Computation, T. Mogensen et al., Eds. LNCS, vol. 2566. Springer, 109-134.

Cousot, P. And Cousot, R. 1992. Abstract interpretation and application to logic programs. J. Logic Programming 13, 2\&3, 103-179.

Cousot, P., Cousot, R., FÄhndrich, M., And Logozzo, F. 2013. Automatic inference of necessary preconditions. In VMCAI'13. LNCS, vol. 7737. Springer, 128-148.

Cousot, P. And Halbwachs, N. 1978. Automatic discovery of linear restraints among variables of a program. In POPL. ACM Press, 84-96.

De Angelis, E., Fioravanti, F., Pettorossi, A., And Proietti, M. 2014. Program verification via iterated specialization. Sci. Comput. Program. 95, 149-175.

De Angelis, E., Fioravanti, F., Pettorossi, A., and Proietti, M. 2014. VeriMAP: A tool for verifying programs through transformations. In TACAS 2014. LNCS, vol. 8413. Springer, $568-574$.

De Angelis, E., Fioravanti, F., Pettorossi, A., And Proietti, M. 2017. Semantics-based generation of verification conditions via program specialization. Sci. Comput. Program. 147, $78-108$.

Dillig, I., Dillig, T., Li, B., And McMillan, K. L. 2013. Inductive invariant generation via abductive inference. In OOPSLA 2013. ACM, 443-456. 
Doménech, J. J., Gallagher, J. P., And Genaim, S. 2019. Control-flow refinement by partial evaluation, and its application to termination and cost analysis. Theory Pract. Log. Program. 19, 5-6, 990-1005.

Dutertre, B. 2014. Yices 2.2. In CAV 2014. LNCS, vol. 8559. Springer, 737-744.

Gallagher, J. P. 2019. Polyvariant program specialisation with property-based abstraction. In VPT 2019. EPTCS, vol. 299. 34-48.

Gallagher, J. P. And Lafave, L. 1996. Regular approximation of computation paths in logic and functional languages. In Partial Evaluation. LNCS, vol. 1110. Springer, 115-136.

Grebenshchikov, S., Lopes, N. P., Popeea, C., And Rybalchenko, A. 2012. Synthesizing software verifiers from proof rules. In PLDI 2012. ACM, 405-416.

Gulavani, B. S., Chakraborty, S., Nori, A. V., and Rajamani, S. K. 2008. Automatically refining abstract interpretations. In TACAS 2008. LNCS, vol. 4963. Springer, 443-458.

Gulwani, S., Jain, S., And Koskinen, E. 2009. Control-flow refinement and progress invariants for bound analysis. In PLDI. ACM, 375-385.

Gupta, A. And Rybalchenko, A. 2009. Invgen: An efficient invariant generator. In $C A V$, A. Bouajjani and O. Maler, Eds. LNCS, vol. 5643. Springer, 634-640.

Gurfinkel, A., Kahsai, T., Komuravelli, A., And Navas, J. A. 2015. The SeaHorn verification framework. In $C A V$ 2015. LNCS, vol. 9206. Springer, 343-361.

Hermenegildo, M. V., Bueno, F., Carro, M., et Al. 2012. An overview of Ciao and its design philosophy. Theory and Practice of Logic Programming 12, 1-2, 219-252.

Howe, J. M., KIng, A., AND Lu, L. 2004. Analysing logic programs by reasoning backwards. In Program Development in Computational Logic. LNCS, vol. 3049. Springer, 152-188.

Jaffar, J., Murali, V., Navas, J. A., And Santosa, A. E. 2012. TRACER: A symbolic execution tool for verification. In $C A V$ 2012. LNCS, vol. 7358. Springer, 758-766.

Jones, N., Gomard, C., And Sestoft, P. 1993. Partial Evaluation and Automatic Software Generation. Prentice Hall.

Kafle, B. and Gallagher, J. P. 2017a. Constraint specialisation in Horn clause verification. Sci. Comput. Program. 137, 125-140.

Kafle, B. And Gallagher, J. P. 2017b. Horn clause verification with convex polyhedral abstraction and tree automata-based refinement. Comput. Lang. Syst. Struct. 47, 2-18.

Kafle, B., Gallagher, J. P., Gange, G., Et Al. 2018. An iterative approach to precondition inference using constrained Horn clauses. Theory Pract. Log. Program. 18, 553-570.

KARR, M. 1976. Affine relationships among variables of a program. Acta Informatica 6, 133-151.

Marriott, K. And SøndergaArd, H. 1993. Precise and efficient groundness analysis for logic programs. ACM Letters Program. Lang. Syst. 2, 1-4, 181-196.

Miné, A. 2006. The octagon abstract domain. High. Order Symb. Comput. 19, 1, 31-100.

Miné, A. 2012. Inferring sufficient conditions with backward polyhedral under-approximations. Electronic Notes in Theor. Comp. Sci. 287, 89-100.

MoY, Y. 2008. Sufficient preconditions for modular assertion checking. In VMCAI 2008. LNCS, vol. 4905. Springer, 188-202.

Muthukumar, K. And Hermenegildo, M. 1990. Deriving A Fixpoint Computation Algorithm for Top-down Abstract Interpretation of Logic Programs. Technical Report ACT-DC-153-90, Microelectronics and Computer Technology Corporation (MCC), Austin, TX 78759. April.

Padhi, S., Sharma, R., And Millstein, T. D. 2016. Data-driven precondition inference with learned features. In PLDI 2016. ACM, 42-56.

Peralta, J. C., Gallagher, J. P., And SaĞLam, H. 1998. Analysis of imperative programs through analysis of constraint logic programs. In $S A S$ 1998. LNCS, vol. 1503. 246-261.

Popeea, C. And Chin, W. 2013. Dual analysis for proving safety and finding bugs. Sci. Comput. Program. 78, 4, 390-411. 
Puebla, G. And Hermenegildo, M. V. 1999. Abstract multiple specialization and its application to program parallelization. J. Log. Program. 41, 2-3, 279-316.

SAnkaranarayanan, S., Sipma, H., And Manna, Z. 2004. Non-linear loop invariant generation using Gröbner bases. In POPL. ACM, 318-329.

Seghir, M. N. And Schrammel, P. 2014. Necessary and sufficient preconditions via eager abstraction. In APLAS 2014. LNCS, vol. 8858. Springer, 236-254. 